\title{
Caregiver Characteristics and Symptoms of Pediatric Bipolar Disorder
}

\author{
BY \\ AMY T. PETERS \\ B.A., Boston College, 2010 \\ M.A., University of Illinois at Chicago, Chicago, 2013
}

THESIS

Submitted as partial fulfillment of the requirements for the degree of Master of Arts in Psychology in the Graduate College of the University of Illinois at Chicago, 2013

Chicago, Illinois

Defense Committee:

Amy West, Chair and Advisor

Ellen Herbener

Dave Henry 


\section{TABLE OF CONTENTS}

CHAPTER

$\underline{\text { Page }}$

I. INTRODUCTION ........................................... 1

A. Background ............................................. 1

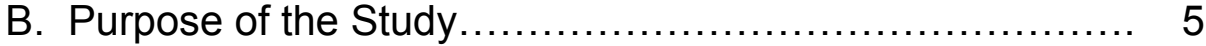

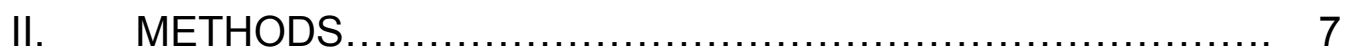

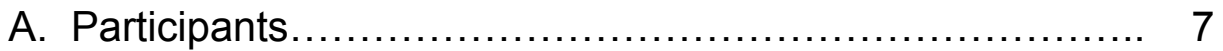

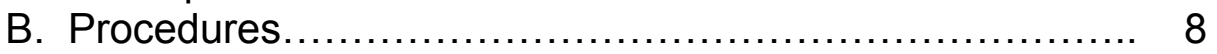

C. Measures................................................ 8

1. Washington University Kiddie Schedule for Affective Disorders and Schizophrenia......................... 8

2. Kiddie Young Mania Rating Scale................... 9

3. Children's Depression Rating Scale.................. 9

4. Child Mania Rating Scale........................... 10

5. Child Bipolar Depression Rating Scale............... 10

6. Coping Health Inventory for Parents................. 10

7. Symptom Checklist 90-R.......................... 11

8. Parental Stress Scale.............................. 12

9. Therapy Outcomes Parent Scale..................... 12

D. Data Analytic Approach.................................... 13

III. RESULTS ..................................................... 14

A. Preliminary Analyses........................................... 14

B. Main Analyses............................................... 17

IV. DISCUSSION............................................... 22

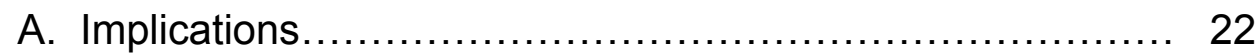

B. Limitations .................................................. 26

C. Conclusion................................................ 27

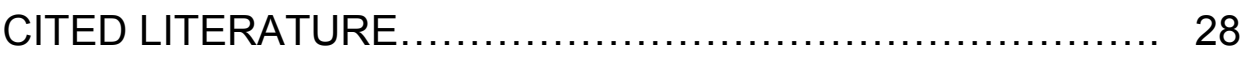

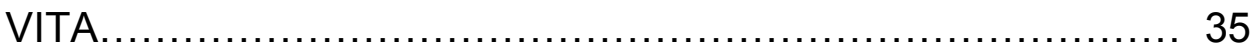




\section{LIST OF TABLES}

$\underline{\text { TABLE }} \quad \underline{\text { Page }}$

I. DEMONGRAPHIC AND CLINICAL CHARACTERISTICS OF 64 CHILDREN AND FAMILIES WITH PEDIATRIC BIPOLAR

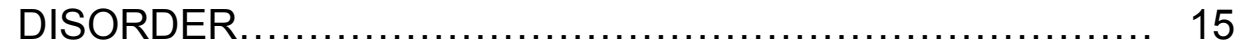

II. DESCRIPTIVE STATISTICS FOR VARIABLES OF INTERST 16

III. ASSOCIATION BETWEEN PARENT CHARACTERISTICS AND SYMPTOMS OF PEDIATRIC BIPOLAR DISORDER .... 18 


\section{LIST OF FIGURES}

FIGURE $\quad$ Page

1. Association between caregiver psychopathology and child depression as a function of parent coping skill use........... 19

2. Association between caregiver psychopathology and child mania as a function of parent coping skill use................. 


\section{LIST OF ABBREVIATIONS}

$\begin{array}{ll}\text { ADHD } & \text { Attention Deficit Hyperactivity Disorder } \\ \text { ANOVA } & \text { One Way Analysis of Variance } \\ \text { CBDRS } & \text { Child Bipolar Depression Rating Scale } \\ \text { CDRS-R } & \text { Children's Depression Rating Scale Revised } \\ \text { CFF-CBT } & \text { Child and Family Focused Cognitive Behavioral Therapy } \\ \text { CMRS } & \text { Child Mania Rating Scale } \\ \text { FFT } & \text { Family Focused Treatment } \\ \text { IFP } & \text { Individual Family Psychoeduation } \\ \text { KYMRS } & \text { Kiddie Young Mania Rating Scale } \\ \text { MFPG } & \text { Multi-family Psychoeducation Group } \\ \text { NOS } & \text { Not Otherwise Specified } \\ \text { PBD } & \text { Pediatric Bipolar Disorder } \\ \text { PSS } & \text { Parental Stress Scale } \\ \text { SD } & \text { Standard Deviation } \\ \text { SCL-90-R } & \text { Symptom Checklist 90-Revised } \\ \text { TOPS } & \text { Therapy Outcomes Parents Scale (TOPS) } \\ \text { WASH-U-KSADS } & \begin{array}{l}\text { Washington University in St. Louis Kiddie Schedule for } \\ \text { Affective Disorders and Schizophrenia }\end{array} \\ \end{array}$




\section{SUMMARY}

The role of family dynamics in symptoms, course, and outcome of pediatric bipolar disorder (PBD) has been extensively studied. Individual caregiver characteristics have received less attention in the literature. Understanding how caregiver traits are related to child symptoms could have important implications for psychosocial treatment of pediatric bipolar disorder, where the parents are heavily involved. This study explored the direct and interactive associations between parent psychopathology, stress, efficacy, and coping and symptoms of pediatric bipolar disorder in 64 parents of children meeting DSM-IV criteria for bipolar I, II, or NOS.

Results most consistently indicated that parent psychopathology predicted increased child mania and depression severity among parents with low, but not average or above average use of coping skills.

Without effective coping skills, parent psychopathology may have significant effects of the severity of their child's symptoms of mania or depression. Interventions which teach caregivers effective tools and skills to cope with their child's illness may mitigate the effects of their own psychopathology and lead to more favorable treatment outcomes. 


\section{Introduction}

\section{A. Background}

Pediatric bipolar disorder (PBD) is a devastating illness characterized by severe periods of episodic mood disturbance. Children with bipolar disorder experience chronic and extreme dysregulation that is associated with significant psychosocial impairment (Goldstein et al., 2009). Specifically, children with bipolar disorder demonstrate academic underperformance, disruptive behavior, poor social skills, and high levels of family stress (Geller et al., 2002). Delays to first treatment of PBD are associated with a chronic and severe course of illness (Post, 1999), whereas early intervention may buffer against negative outcomes (Berk et al., 2010). Pharmacological agents are the first line of treatment for PBD, but their use alone fails to bring children to full, sustained remission (Washburn, West, \& Heil, 2011). As such, psychosocial interventions have been developed as an adjunct to pharmacotherapy to address the complex constellation of symptoms and associated social, cognitive, and family difficulties in PBD.

Family-based psychosocial interventions, especially, are warranted for PBD given the sensitive developmental period of its onset. Relative to their counterparts with adult onset, children with bipolar disorder are more likely to experience rapid cycling, mixed mood states, demonstrate high rates of comorbidity, as well as experience more severe mood symptoms and less time euthymic during adulthood (Geller, Tillman, Bolhofner, \& Zimerman, 2008; Geller, Tillman, Craney, \& Bolhofner, 2004). Moreover, despite the severity of their 
condition, children have compromised insight and understanding of their own psychopathology, and tend to underreport their symptoms (Youngstrom, Findling, \& Calabrese, 2004). The accumulation of negative prognostic indicators associated with PBD places an enormous burden on the caretakers of affected children to help intervene during this period of significant risk. Parents, therefore, bear a great responsibility to help their children identify and monitor symptoms and seek treatment. They are integral to the management of symptoms and achieving optimal functioning for the patient and whole family. Accordingly, psychosocial interventions that include the family are necessary for the comprehensive management of the disorder. The development of psychosocial interventions specific to PBD is a relatively new area of work, but the familybased interventions currently being developed and tested in childhood and adolescence have yielded significant reductions in mood symptoms and improvements in psychosocial functioning thus far (West \& Pavuluri, 2009).

Family relationships, however, are not necessarily ipso facto protective factors (Miklowitz, 2007). Evidence from studies of expressed emotion suggest that the specific quality of family environment has important implications for understanding the development and maintenance of mood symptoms (Miklowitz, 2004). Overall, families of patients affected by mood disorders and schizophrenia have high levels of expressed emotion, which is associated with relapse (Butzlaff \& Hooley, 1998; Miklowitz, 2004). Consistent with these findings, families of children with PBD have low levels of cohesion, expressiveness, and activity, and high levels of conflict and expressed emotion (Belardinelli et al., 2008; Schenkel, 
West, Harral, Patel, \& Pavuluri, 2008; Sullivan \& Miklowitz, 2010). These deficits in family functioning are associated with poor illness course and adverse treatment outcomes for youth. One study established expressed emotion as a predictor of time to recovery in depressed children (Asarnow, Goldstein, Tompson, \& Guthrie, 1993) and another as a predictor of symptom severity among bipolar adolescents (Miklowitz, Biuckians, \& Richards, 2006). Studies of both pharmacological and psychosocial interventions in bipolar children and adolescents demonstrate that poor family functioning moderates treatment outcome, such that patients of families with impaired functioning benefit less from treatment or require a more intensive intervention to achieve symptomatic improvement (Miklowitz et al., 2009; Sullivan, Judd, Axelson, \& Miklowitz, 2012; Townsend, Demeter, Youngstrom, Drotar, \& Findling, 2007).

Viewed through this lens, child-focused treatment alone may not be sufficient to achieve improvements in mood symptoms and family functioning. For that reason, several existing interventions work intensively with the parents alongside work with the child, under the expectation that caregiver focused aspects of intervention will help to achieve optimal functioning for the whole family and facilitate improvements in mood symptoms. Despite their different theoretical underpinnings, family based interventions for children with PBD such as child and family focused cognitive behavioral therapy (CFF-CBT) and individual family or multi-family group psychoeduation (IFP; MFPG) do so through common strategies such as providing parents with education about PBD and the impact parent and family systems have on its course of illness, boosting 
parenting efficacy, helping parents cope with the illness burden of caring for a child with bipolar disorder, and managing the parents own psychological needs (Fristad, 2006; West \& Weinstein, 2012). Similar parent-oriented strategies are also utilized in interventions designed for adolescents with bipolar disorder, such as family-focused treatment (FFT) (FFTMiklowitz et al., 2008) and dialectical behavioral treatment (DBT) (Goldstein, Axelson, Birmaher, \& Brent, 2007).

Although these interventions are designed to accommodate caregiver needs expected to be involved in the child's illness, there is actually a limited evidence base to fundamentally explain which and how these specific parent level factors relate to child mood symptoms. In contrast to the large body of research investigating the role of family factors in illness severity, few studies have examined how specific parent characteristics relate to child symptoms. A few studies have looked at parent factors found that compared to controls and youth with ADHD, parent-child relationships in the PBD group were characterized by significantly less warmth and greater tension and hostility (Geller et al., 2000) and that longitudinally, lower maternal warmth in the PBD group significantly predicted shorter time to relapse after recovery from mania at both the 2 and 4 year follow up (Geller et al., 2002; Geller et al., 2004).

Additionally, in a separate study of adolescents with unipolar depression and bipolar disorder, Robertson, Kutcher, Bird, \& Glasswick (2001) found that bipolar adolescents reported more problems with parents compared to depressed adolescents and healthy controls (Robertson, Kutcher, Bird, \& Grasswick, 2001). 
Indeed, these findings regarding parental warmth and conflict have important treatment implications. However, to achieve optimal implementation of the parent-focused aspects of psychosocial interventions for PBD, a more complete understanding of exactly how caregiver characteristics are implicated in the maintenance of PBD symptoms is needed. Ultimately, a comprehensive understanding of these relationships could help guide flexible implementation and adaptation of the parent-focused aspects of psychosocial interventions to better serve specific needs of the child and family.

\section{B. Purpose of the Study}

This study builds on previous work regarding the family context in pediatric bipolar disorder by examining how caregiver characteristics are involved in the symptomatic status of children with bipolar disorder. The aim of the present study is to gain a better understanding of the relation between parent traits and mood symptoms in youth, and to describe the impact of parent behaviors on child mood state. Specifically, we are interested in how parent psychopathology, coping skills, stress and burden of caring for a child with bipolar disorder, and parent knowledge and efficacy about parenting a child with bipolar disorder are related to child symptoms of mania and depression. We seek to assess both the direct influence of parent psychopathology, parenting stress, coping and parenting efficacy on symptoms of depression and mania in youth, as well as whether skills that can be taught to parents through psychosocial intervention (i.e., coping skills 
and parenting efficacy) may buffer against the severity of child mood symptoms for caregivers higher in psychopathology and stress. 


\section{Methods}

\section{A. Participants}

Participants $(n=64)$ for the study were referred from providers in the Pediatric Mood Disorders Clinic at the University of Illinois at Chicago Medical Center. All participants were part of a randomized clinical trial of a family-based psychosocial treatment for pediatric bipolar disorder. A trained research assistant assessed potential participants for eligibility. All eligible participants fulfilled the following criteria: a DSM-IV diagnosis of bipolar I, II, or not otherwise specified (NOS) based on the Washington University in St. Louis Kiddie Schedule for Affective Disorders and Schizophrenia (WASH-U-KSADS) (Geller et al., 2001) interview with the child and at least one parent; stable on current medication regimen; age between $7-13$ years. Participants were not eligible for the study if the parent(s) met criteria for an acute episode of mania or depression, or if the child scored $<70$ on the Kaufman Brief Intelligence Test, Second Addition (Kaufman, 2004), was actively psychotic, acutely suicidal, reported active substance use, or suffered from any neurological or other medical condition that would potentially cause or complicate presenting psychiatric symptoms.

The average age of the sample was 9.21 years $(S D=1.56)$. Forty-six percent were female; 56\% European Caucasian, 30\% African American, 5\% Hispanic, 5\% American Indian or Alaskan, 2\% Native American or Pacific Islander, and $2 \%$ identified as "Other." Thirty-six percent of the sample was diagnosed with bipolar I, 3\% bipolar II, and $61 \%$ with bipolar disorder not 
otherwise specified. Thirty percent had a co-morbid anxiety disorder, $78 \%$ had co-morbid ADHD, 37\% had co-morbid oppositional defiant disorder, and 10\% had co-morbid conduct disorder. Nearly half of the sample (42\%) reported their current or most recent episode as mixed, $27 \%$ reported manic or hypomanic, $10 \%$ depressed, and $28 \%$ did not specify.

\section{B. Procedures}

After the informed consent procedures, youth with suspected or diagnosed bipolar spectrum disorder and their caregiver(s) were screened for eligibility for the study. The child and parent(s) were interviewed separately by trained interviewers using the Washington University Kiddie Schedule for Affective Disorders and Schizophrenia (WASH-U-KSADS)(Geller et al., 2001). After confirmation of a bipolar spectrum disorder diagnosis and the administration of inclusion/exclusion measures, youth and caregivers completed a battery of baseline assessments and questionnaires, including measures of symptoms, global functioning, and child, caregiver, and family psychosocial functioning. The current study uses baseline clinician and parent-rated measures of child depression and mania symptoms, and parent self-report of coping strategies, psychopathology, stress, and parenting efficacy.

\section{Measures}

1. Washington University Kiddie Schedule for Affective Disorders and Schizophrenia (WASH-U-KSADS) 
The WASH-U-KSADS (Geller et al., 1996) is a semi-structured clinician administered interview designed to yield a DSM-IV diagnosis (American Psychiatric Association, 1994). This diagnostic instrument has demonstrated good test-retest reliability and inter-rater reliability (Geller et al., 2001b).

\section{Kiddie Young Mania Rating Scale (K-YMRS)}

The K-YMRS is an eleven-item, multiple-choice rating scale used to assess the severity of manic symptoms in children. The scale was originally developed for the evaluation of adult patients suffering from bipolar disorder (Young, 1978). It has since been modified for use in pediatric patients and is established as a reliable and valid measure of manic symptoms in children ages 5-17 (Fristad, Weller, \& Weller, 1992; Fristad, Weller, \& Weller, 1995; Youngstrom, Danielson, Findling, Gracious, \& Calabrese, 2002). Scores are calculated by summing across the 11 items. The internal consistency of the K-YMRS in this sample was .60.

\section{Children's Depression Rating Scale Revised (CDRS-R)}

The CDRS-R (E. Poznanski, 1984) is a reliable and valid clinicianrated instrument for measuring the severity of depression in children. The CDRS-R resembles the Hamilton Depression Rating Scale in its use of categories and subcategories (Hamilton, 1967), but with developmental modifications that are more appropriate for a pediatric population. The scale has demonstrated strong inter- 
rater reliability and validity (E. O. Poznanski, Cook, \& Carroll, 1979). Scores are calculated by summing scores across the 17 items that assess depressive symptoms, each of which are rated on a 5 point Likert-type scale. The CDRS demonstrated good internal consistency in this sample $(\alpha=.83)$.

\section{Child Mania Rating Scale (CMRS)}

The CMRS parent report (M. N. Pavuluri, Henry, Devineni, Carbray, \& Birmaher, 2006) measures symptoms of mania, such as elevated mood, increased motor activity, hypersexual interest, lack of sleep, irritability, tangential language and pressured speech, and aggressive behavior, with high inter-rater reliability and concurrent validity with other mania rating scales. Scores are calculated by summing the 21 items assessing mania, each of which are rated on a Likert scale ranging from 0 (never) to 3 (very often). The CMRS demonstrated good internal consistency in this sample $(\alpha=.89)$.

\section{Child Bipolar Depression Rating Scale (CBDRS)}

The CBDRS (M. Pavuluri, Unpublished) parent report consists of content valid questions concerning childhood depression symptoms. Scores are calculated by summing 22 items assessing depression, each of which are rated on a Likert scale ranging from 0 (never) to 3 (very often). The CBDRS demonstrated good internal consistency in this sample $(\alpha=.87)$.

\section{Coping Health Inventory for Parents (CHIP)}


The CHIP (McCubbin \& Thompson, 1991) is specifically designed to measure parents' responses to management of family life when they have a child who is seriously or chronically ill. It includes subscales measuring family integration and cooperation, social support and psychological stability, and understanding of the illness through communication with professionals. Scores are calculated by summing scores for the total number of items, and the item in each of the subscales, which are each rated on a Likert scale from 0 (not helpful) to 3 (extremely helpful). All subscales and overall scale have demonstrated high internal consistency. The CDRS demonstrated good internal consistency in this sample $(\alpha=.80)$.

\section{Symptom Checklist 90-R (SCL-90-R)}

The SCL-90-R (Derogatis, 2000) is a brief instrument with extensive data on its reliability and validity that helps evaluate a broad range of psychological problems and symptoms of psychopathology in adults. It contains 90 items rated on a 5-point scale. The measure yields nine primary symptom dimensions (Somatization, Obsessive-Compulsive, Interpersonal Sensitivity, Anxiety, Depression, Hostility, Phobic Anxiety, Paranoid Ideation, and Psychoticism) and three global indices (Global Severity, Positive Symptom Index, Positive Symptom Total). This study utilizes the Global Severity index, which reflects both the frequency and severity of parent symptoms reported. The SCL-90-R 
demonstrated excellent internal consistency in this sample $(\alpha=$ $.98)$.

\section{Parental Stress Scale (PSS)}

The PSS (Berry \& Jones, 1995) assesses caregivers feelings regarding the parent-child relationship, such as feeling overwhelmed, satisfied, close, and worried. Scores on the PSS are calculated by summing scores across 18 items, each of which are rated on a Likert scale ranging from 1 (strongly disagree) to 5 (strongly agree). The scale has good test-retest reliability and has been standardized in several populations. Validity was established through the scale's correlation with other well-established measures of parenting stress, such as the Parenting Stress Index (PSI) (Abidin, 1986). The internal consistency of the PSS in this sample was .50 .

\section{Therapy Outcomes Parents Scale (TOPS)}

The TOPS was developed to assess parent feelings and perceptions regarding their child's bipolar disorder, including their knowledge about the disorder and sense of efficacy in coping with it. Parents' rate 20 items on a 5-point Likert scale ranging from 'strongly disagree' to 'strongly agree.' Higher scores indicate greater knowledge and perceived self-efficacy in managing the child's disorder. This face-valid measure has shown good internal reliability (The CDRS demonstrated good internal consistency in 
this sample $(\alpha=.88)$ in previous studies (West et al., 2009). The TOPS demonstrated acceptable internal consistency in this sample $(\alpha=.76)$.

\section{Data Analytic Approach}

The present study utilized multiple linear regression models in SPSS 20.0 to model the effects of caregiver characteristics on child mood symptoms. Models were conducted separately to examine the effects of each parent baseline characteristic on child mood symptoms, including effects for the focal predictor (parent psychopathology, parenting stress), moderator variable (parent coping, parenting efficacy), and predictor by moderator. Models were conducted using both parent-reported and clinician-rated/child reported outcome measures of mood symptoms, as previous literature has indicated inconsistencies in symptom report can exist according to informant (De Los Reyes, Youngstrom, Pabon, et al., 2011; De Los Reyes, Youngstrom, Swan, et al., 2011; Youngstrom et al., 2004; Youngstrom et al., 2011). Effects for demographic controls (age, gender), were included in models when demographic characteristics demonstrated significant associations with dependent variables in preliminary analyses. Regression analyses involved the hierarchical entry of demographic controls, focal predictor, moderator variable, and interactions between the focal predictor and proposed moderator. All continuous independent variables were mean-centered. The only categorical predictor, sex, was dummy coded with females as the comparison level. To probe the form of the interaction terms, simple slopes of the focal predictor on child mood symptoms were tested at the 
mean, and one standard deviation (SD) above and below the mean of the moderator variables, creating "low" (1 SD below the mean), "moderate" (at the mean), and "high" (1 SD above the mean) levels of the moderator variable (Aiken \& West, 1991). 


\section{Results}

\section{A. Preliminary Analyses}

All variables were first checked for normal distribution. This inspection revealed that parent psychopathology was a skewed variable. We took the natural logarithm of caregiver psychopathology to normalize the distribution, and used this logarithmic transformation in all further analyses.

Demographic and clinical characteristics of the total sample are reported in Table I.

Table I. Demographic and clinical characteristics of 64 children and families with pediatric bipolar disorder

\begin{tabular}{lll}
\hline & Mean & SD \\
\hline Age & 9.21 & 1.56 \\
\hline Sex (female) & $\mathbf{N}$ & $\%$ \\
\hline Family Income (<50, 000) & 29 & 46 \\
\hline Ethnicity & 22 & 38 \\
\hline$\quad$ & \\
\hline European Caucasian & 36 & 56 \\
\hline African American & 19 & 30 \\
\hline Hispanic & 3 & 5 \\
\hline Native American or Pacific Islander & 1 & 2 \\
\hline American Indian or Alaskan & 3 & 5 \\
\hline Other & 1 & 2 \\
\hline Diagnosis & & \\
\hline$\quad$ Bipolar I & 23 & 36 \\
\hline$\quad$ Bipolar II & 2 & 3 \\
\hline Bipolar NOS & 39 & 61 \\
\hline Co-morbidities & & \\
\hline Anxiety Disorder & 19 & 30 \\
\hline Attention Deficit Hyperactivity Disorder & 50 & 78 \\
\hline Oppositional Defiant Disorder & 23 & 37 \\
\hline Conduct Disorder & 6 & 10 \\
\hline Current or Most Recent Episode &
\end{tabular}

Current or Most Recent Episode 


\begin{tabular}{lll}
\hline Manic/hypomanic & 16 & 27 \\
\hline Depressed & 6 & 10 \\
\hline Mixed & 25 & 42 \\
\hline Unspecified & 17 & 28 \\
\hline $\begin{array}{l}\text { *Percentages are represented as } \mathrm{n}(\%) \\
\text { number of available cases }\end{array}$ &
\end{tabular}

Descriptive statistics for all study measures are reported in Table II.

Table II. Descriptive statistics for variables of interest; $n=64$

\begin{tabular}{lll}
\hline Independent Variables & Mean & SD \\
\hline$S C L$ & -1.28 & 1.19 \\
\hline$P S S$ & 44.39 & 11.48 \\
\hline CHIP & 81.75 & 24.25 \\
\hline TOPS & 71.16 & 10.94 \\
\hline Dependent Variables & & \\
\hline CMRS & 23.66 & 10.45 \\
\hline YMRS & 12.14 & 5.48 \\
\hline CBDRS & 19.01 & 9.71 \\
\hline CDRS & 41.25 & 10.92
\end{tabular}

Bivariate correlations and one-way analysis of variance (ANOVA) were used to examine associations between demographic factors (e.g., child age, child sex, child race, family income) and outcome measures of mania and depression. Child age positively predicted parent-rated depressive symptom severity $(r=.26$, $p=.047)$, and females demonstrated higher depressive symptom severity relative to males, $F(1,59)=3.88, p=.05$. Therefore, in models predicting parent- 
rated depressive symptom severity, child age and sex were included as control variables. Race and income were unrelated to parent rated depressive symptom severity (all p's > .602). Age, sex, race, and income were not associated with parent-rated mania symptoms, or child-reported/clinician-rated symptoms of mania or depression (all p's > .161).

Bivariate correlations were also used to examine associations between predictor variables (e.g. parent psychopathology, parenting stress, parent coping, and parenting efficacy). Parenting efficacy and parenting stress were correlated, $r=-.45, p<.001$. No other associations between predictor variables were significant (all p's > .069).

\section{B. Main Analyses: Effects of Parent Characteristics on Child Mood}

\section{Symptoms}

Results of the models, including parameter estimates, standard error, and t-values intervals are presented in Table III. A model predicting parent-reported child depressive symptoms indicated a significant interaction between parent psychopathology and coping, as is illustrated in Figure 1. Increases in the log of parent psychopathology predicted increases in child depressive symptoms for parents low in coping, $b=3.08, S E=1.45, t(2.13), p=.038$. Parent psychopathology was unrelated to child depressive symptoms among parents with moderate, $b=.45, S E=.98, t(.46), p=.65$, or high, $b=-2.18, S E=1.44$, $t(-1.52), p=.130$ use of coping skills. This model returned no main effects of age, sex, parent psychopathology, or coping. 
Table III. Association between parent characteristics and symptoms of pediatric bipolar disorder; $n=64$

\begin{tabular}{|c|c|c|c|c|c|c|c|c|c|c|c|c|}
\hline \multirow{2}{*}{$\begin{array}{l}\text { PARENT CHARACTERISTICS } \\
\text { Psychopathology (SCL-90- } \\
\text { R) }\end{array}$} & \multicolumn{3}{|c|}{$\begin{array}{c}\text { Effect on Parent-Rated } \\
\text { Depression }\end{array}$} & \multicolumn{3}{|c|}{$\begin{array}{c}\text { Effect on Parent- } \\
\text { Rated Mania }\end{array}$} & \multicolumn{3}{|c|}{$\begin{array}{c}\text { Effect on Child- } \\
\text { Rated Depression }\end{array}$} & \multicolumn{3}{|c|}{$\begin{array}{c}\text { Effect on Child-Rated } \\
\text { Mania }\end{array}$} \\
\hline & $\mathrm{b}$ & SE & $\mathrm{t}$ & $\mathrm{b}$ & SE & $\mathrm{t}$ & $\mathrm{b}$ & SE & $\mathrm{t}$ & $\mathrm{b}$ & SE & $t$ \\
\hline Age & 1.32 & .80 & 1.66 & - & - & - & - & - & - & - & - & - \\
\hline Sex & 3.40 & 2.49 & 1.37 & - & - & - & - & - & - & - & - & - \\
\hline Psychopathology & .45 & .98 & .46 & .45 & 1.09 & .42 & -.20 & 1.19 & -.17 & -.15 & .59 & -.25 \\
\hline Coping & -.05 & .06 & -.84 & $<.01$ & .06 & .06 & .06 & .07 & .86 & $.07^{*}$ & .03 & 1.96 \\
\hline Psychopathology x Coping & $-.12^{*}$ & .05 & -2.50 & $-.13^{*}$ & .05 & -2.37 & -.01 & .06 & -.06 & .01 & .03 & .29 \\
\hline Age & 1.38 & .76 & 1.83 & - & - & - & - & - & - & - & - & - \\
\hline Sex & 3.71 & 2.52 & 1.47 & - & - & - & - & - & - & - & - & - \\
\hline Psychopathology & -.17 & 1.03 & -.16 & -.23 & 1.19 & -.20 & .23 & 1.25 & .19 & -.21 & .64 & -.34 \\
\hline Parenting Efficacy & -.05 & .11 & -.41 & -.09 & .13 & -.65 & .20 & .14 & 1.43 & -.03 & .07 & -.48 \\
\hline $\begin{array}{l}\text { Psychopathology x } \\
\text { Parenting Efficacy }\end{array}$ & .13 & .09 & 1.37 & .09 & .10 & .93 & .07 & .11 & .66 & -.03 & .05 & -.56 \\
\hline \multicolumn{13}{|l|}{ Parenting Stress (PSS) } \\
\hline Age & 1.13 & .82 & 1.37 & - & - & - & - & - & - & - & - & - \\
\hline Sex & 4.80 & 2.55 & 1.88 & - & - & - & - & - & - & - & - & - \\
\hline Parenting Stress & -.15 & .11 & -1.42 & -.03 & .12 & -.21 & -.16 & .12 & -1.31 & .04 & .06 & .59 \\
\hline Coping & .01 & .06 & .11 & .04 & .06 & .68 & .09 & .07 & 1.31 & $.07^{*}$ & .03 & 2.24 \\
\hline Parenting Stress x Coping & .01 & .01 & 1.38 & -.01 & .01 & -.20 & .01 & .01 & 1.37 & $<.01$ & $<.01$ & 1.04 \\
\hline Age & 1.36 & .74 & 1.83 & - & - & - & - & - & - & - & - & - \\
\hline Sex & 4.47 & 2.41 & 1.86 & - & - & - & - & - & - & - & - & - \\
\hline Parenting Stress & -.14 & .11 & -1.28 & -.07 & .13 & -.53 & -.03 & .14 & .83 & .07 & .08 & .83 \\
\hline Parenting Efficacy & -.10 & .12 & -.86 & -.08 & .14 & -.61 & .17 & .14 & .24 & .04 & .07 & .57 \\
\hline $\begin{array}{l}\text { Parenting Stress x } \\
\text { Parenting Efficacy }\end{array}$ & .01 & .01 & 1.57 & $<.01$ & .01 & .12 & .01 & .01 & .25 & .01 & .09 & .15 \\
\hline
\end{tabular}


Figure 1. Association between caregiver psychopathology and child depression as a function of parent coping skill use

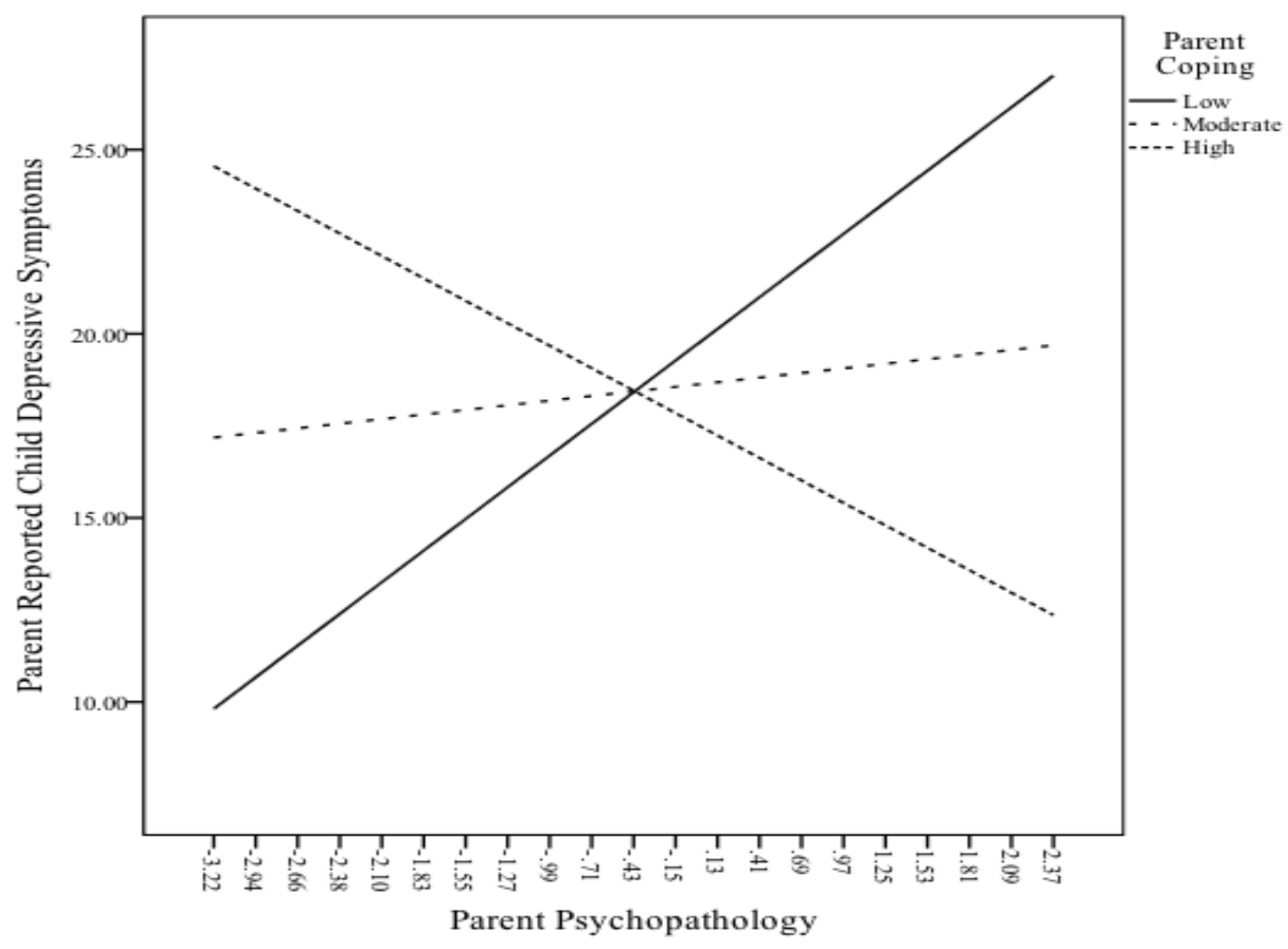

The same pattern of results was observed for parent-rated child manic symptoms. There was again a significant interaction between parent psychopathology and coping, as is illustrated in Figure 2. Increases in the log of parent psychopathology most strongly predicted increases in child manic symptoms for parents low in coping, $b=3.25, S E=1.62, t(2.01), p=.049$. There was no association between parent psychopathology and child manic symptoms for parents with moderate, $b=.45, S E=1.09, t(.42), p=.679$, or high use of coping skills, $b=-2.34, S E=1.60, t(-1.46), p=.149$. This model returned no main effects of age, sex, parent psychopathology, or coping. 
Figure 2. Association between parent psychopathology and child mania as a function of parent coping skill use

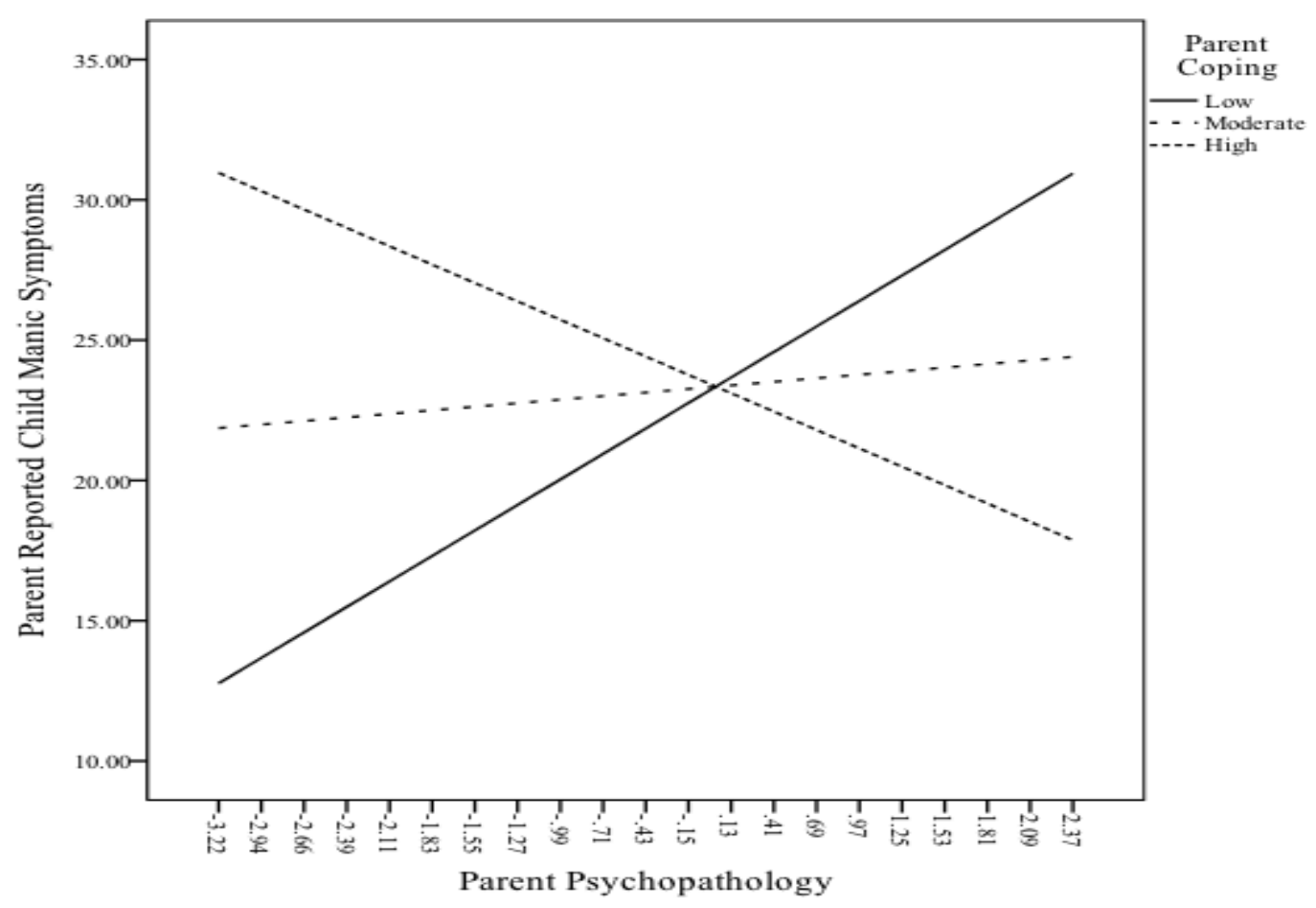

When these models were conducted using clinician-rated/child-reported measures of depressive and manic symptoms, the effect of parent psychopathology did not depend on the level of parent coping (See Table III). Rather, these models revealed a direct association between parent coping and clinician rated symptoms of child mania, such that greater parent use of coping skills was associated with more severe child manic symptoms. That is, increases in parent coping were associated with increases in mania severity. 
In contrast to the findings found above for the effects of parent psychopathology and coping on symptoms of bipolar disorder in children, we did not find any evidence for a relationship between parenting stress or parenting efficacy and child mood symptoms. Models assessing how parenting stress and parenting efficacy relate to child mood symptoms returned no main or interactive effects neither for parent-reported symptoms of child depression and mania, nor for depression and mania symptom ratings gathered from the child by a clinician (See Table III). 


\section{A. Implications}

\section{Discussion}

The present study investigated how parent characteristics relate to the symptomatic profile of youth with bipolar disorder. Family dynamics - as related to the symptoms, course, and outcome in PBD - have been extensively studied. However, individual caregiver characteristics have not received as much attention in the literature. This study provides evidence that individual traits of caregivers may be associated with the experience of symptoms of depression and mania among youth with PBD. We found that parent psychopathology predicted increased child mania and depression severity among parents with low, but not average or above average use of coping skills when rated by parents. In addition we found a direct association between poorer parent coping and increased child mania severity when children were evaluated by clinicians. In contrast, we did not find any evidence that parenting stress or parenting efficacy were related to mood symptoms among bipolar youth.

These findings suggest that, although parent psychopathology does not directly predict symptoms of depression or mania among youth, it is strongly related to symptom severity in parents who report rare or insufficient use of skills to cope with their child's affective illness. Previous research has suggested that the role of parent psychopathology in a child's mental illness may operate through two different pathways (Neiderhiser, Reiss, Hetherington, \& Plomin, 1999). First, children of parents with psychological disturbance may have a genetic predisposition to develop symptoms of their own. Second, children, especially young children of a pre-pubertal age, may be viewed as a "sponge," 
modeling behaviors they observe in their parents. When parents are emotionally distressed, the traits and behaviors their children learn may be maladaptive and contribute to the development of psychopathology. Remarkably, findings from this study suggest that parents who employ skills to cope with their child's illness may have the power to mitigate these negative trajectories, as our findings indicate that the effects of parent psychopathology on child mood symptoms are lessened when parents use skills to cope with their child's illness.

It was interesting that although the association between parent psychopathology and child symptoms of depression and mania depended on the level of coping when models were conducted using parent-rated outcomes, we observed a direct association between coping and manic severity using clinicianrated outcomes of youth-report. The direction of this association indicated that increases in parent coping were actually associated with increases in manic symptom severity. Given the cross sectional design of the study, and the potential bi-directional nature of parent and child variables, it is possible that children with a severely manic presentation are more difficult to manage, and in turn, cause their parents to utilize more coping strategies. However, it is not clear why this association would not extend to parent perceptions of the association between coping and their child's symptoms. In general, previous literature has indicated that, especially among this pre-pubertal age group, parent report of child symptoms are more valid and reliable relative to other informants (De Los Reyes, Youngstrom, Pabon, et al., 2011; De Los Reyes, Youngstrom, Swan, et al., 2011; Youngstrom et al., 2004; Youngstrom et al., 2011). This is true even 
when the primary caregiver experiences mood symptoms (Youngstrom et al., 2011). Therefore, we are inclined to more heavily weigh our findings from parentrated outcomes. However, it remains a distinct possibility that the parent-rated outcomes were subject to bias from their own psychopathology, stress, or other traits. More carefully examining the associations between parent coping and child symptoms using additional informants (other family members, teachers, etc.) may help to clarify the directional nature of these associations.

It was surprising that we did not find any evidence to support an association between parenting stress or parenting efficacy and symptoms of pediatric bipolar disorder. A potential explanation is that although parenting stress and parenting efficacy are not involved in the maintenance of symptoms, they may be involved in other aspects surrounding the child's illness, such as problem behaviors or family functioning. Therefore, it would be worthwhile to assess the impact parenting stress and parenting efficacy have on areas of functioning that are often impaired in youth with bipolar disorder, such as school performance, social skills, family functioning, etc. It is also possible that we had a limited statistical ability in our sample to detect an association between parent stress or efficacy and symptoms. For instance, the internal consistency of the parenting stress measure was low relative to the other scales in this study and may have affected our power to detect an association with symptoms.

Furthermore, our measure of parenting efficacy, has traditionally been used as an outcome measure, assessing change after treatment (West et al., 2009). At baseline, this measure showed moderately less variability in scores. Therefore, it 
is possible that this scale is better suited for detecting change than capturing dynamic associations with other variables at first measurement.

Collectively, these results have important implications for psychosocial intervention. On a most basic level, our findings indicate that several parent factors are indeed related to child mood symptoms. Such results argue for the importance of family-based intervention - it is not only child, but also parent traits that are involved in the maintenance of mood symptoms in youth and thus, interventions that address child, but not parent dysfunction may be considered incomplete or insufficient. More specifically, these results suggest that within the context of family-based treatment, it is important not only to devote time in sessions to the child and to the child and parent together, but also to set aside time for parent only sessions. Incorporating parent only sessions allows the time and resources for parents to develop strategies and strengths to recognize and defend against the negative impact their individual behaviors may have on their child's illness. Additionally, although we do not mean to suggest that addressing parenting stress, teaching parents effective parenting methods, or educating parents about the course of bipolar disorder are not important components of parent-focused intervention, nor do we mean to deny the potential role of these parent factors in the child's illness, our results do suggest that improving parent coping skills may be a particularly direct and effective intervention strategy to achieve symptomatic improvement among children whose parents report high levels of their own psychopathology. 


\section{B. Limitations}

Several study limitations are worth noting. First, the cross-sectional design limits conclusions about the direction of the reported associations. It is not only possible, but likely, that the severity of child mood symptoms may contribute to more distressed parent-child relationships, which in turn may exacerbate symptoms of parent psychopathology or cause parents to utilize more coping strategies to manage their child's severe illness. One prospective study does support that parent characteristics predict symptomatic course and outcome in pre-pubertal youth with bipolar disorder. This 8-year follow up found that lower maternal warmth predicted a faster relapse following recovery from a manic episode (Geller et al., 2004). However, more longitudinal studies examining the temporal relationships between changes in parent functioning and long-term outcomes children with bipolar disorder are needed to disentangle whether disturbances in parent functioning are a cause or result of pediatric mania and depression. Equally possible is that child symptoms are a product of a dynamic interaction between parent and child traits - reciprocally affecting one another.

In addition, all of the families enrolled in this study were seeking an intensive family-based psychosocial treatment. It is possible that participants were seeking family-based treatment because of parent-child discord or because of the caregivers' difficulties with managing their child with bipolar disorder or their own symptoms and distress, and therefore the sample may be biased according to the degree of impairment and distress endorsed by parents. Lastly, although the present study utilized a general domain of parent psychopathology, 
we did not collect parental psychiatric diagnoses using a structured interview, making it difficult to assess how specific kinds of parent symptoms relate to youth mood state. It is possible that certain parental diagnoses contribute to child mood symptoms more or less than others.

\section{Conclusion}

These limitations notwithstanding, the findings from this study have important implications for the assessment and treatment of mood symptoms in youth with bipolar disorder. Managing a child's chronic psychiatric illness requires a great deal of coping on behalf of the parent. In the absence of effective coping skills, a parent's own psychopathology may have significant effects of the severity of their child's symptoms of mania or depression. However, interventions that give parents effective tools and skills to cope with their child's illness may mitigate the effects of their own psychopathology and lead to more favorable treatment outcomes. 


\section{CITED LITERATURE}

Abidin, R.R. . (1986). Parenting Stress Index Manual (2nd edn). Charlottesville, VA: Pediatric Psychology Press.

Aiken, L. S., \& West, S. G. . (1991). Multiple regression: Testing and interpreting interactions. : Sage Publications, Incorporated.

Asarnow, J. R., Goldstein, M. J., Tompson, M., \& Guthrie, D. (1993). One-year outcomes of depressive disorders in child psychiatric in-patients: evaluation of the prognostic power of a brief measure of expressed emotion. J Child Psychol Psychiatry, 34(2), 129-137.

Belardinelli, C., Hatch, J. P., Olvera, R. L., Fonseca, M., Caetano, S. C., Nicoletti, M., ... Soares, J. C. (2008). Family environment patterns in families with bipolar children. J Affect Disord, 107(1-3), 299-305. doi: 10.1016/j.jad.2007.08.011

Berk, M., Hallam, K., Malhi, G. S., Henry, L., Hasty, M., Macneil, C., . . McGorry, P. D. (2010). Evidence and implications for early intervention in bipolar disorder. J Ment Health, 19(2), 113-126. doi:

$10.3109 / 09638230903469111$

Berry, Judy O., \& Jones, Warren H. (1995). The Parental Stress Scale: Initial psychometric evidence. Journal of Social and Personal Relationships, 12(3), 463-472.

Butzlaff, R. L., \& Hooley, J. M. (1998). Expressed emotion and psychiatric relapse: a meta-analysis. Arch Gen Psychiatry, 55(6), 547-552. 
De Los Reyes, A., Youngstrom, E. A., Pabon, S. C., Youngstrom, J. K., Feeny, N. C., \& Findling, R. L. (2011). Internal consistency and associated characteristics of informant discrepancies in clinic referred youths age 11 to 17 years. J Clin Child Adolesc Psychol, 40(1), 36-53. doi: $10.1080 / 15374416.2011 .533402$

De Los Reyes, A., Youngstrom, E. A., Swan, A. J., Youngstrom, J. K., Feeny, N. C., \& Findling, R. L. (2011). Informant discrepancies in clinical reports of youths and interviewers' impressions of the reliability of informants. J Child Adolesc Psychopharmacol, 21(5), 417-424. doi: 10.1089/cap.2011.0011

Derogatis, L.R. (2000). Symptom Checklist-90-Revised In A. P. Association (Ed.), Handbook of psychiatric measures. (pp. 81-84).

Fristad, M. A. (2006). Psychoeducational treatment for school-aged children with bipolar disorder. Dev Psychopathol, 18(4), 1289-1306. doi: $10.1017 / \mathrm{S} 0954579406060627$

Fristad, M. A., Weller, E. B., \& Weller, R. A. (1992). The Mania Rating Scale: can it be used in children? A preliminary report. J Am Acad Child Adolesc Psychiatry, 31(2), 252-257. doi: 10.1097/00004583-199203000-00011

Fristad, M. A., Weller, R. A., \& Weller, E. B. (1995). The Mania Rating Scale (MRS): further reliability and validity studies with children. Ann Clin Psychiatry, 7(3), 127-132.

Geller, B., Bolhofner, K., Craney, J. L., Williams, M., DelBello, M. P., \& Gundersen, K. (2000). Psychosocial functioning in a prepubertal and early 
adolescent bipolar disorder phenotype. J Am Acad Child Adolesc

Psychiatry, 39(12), 1543-1548. doi: 10.1097/00004583-200012000-00018

Geller, B., Craney, J. L., Bolhofner, K., Nickelsburg, M. J., Williams, M., \& Zimerman, B. (2002). Two-year prospective follow-up of children with a prepubertal and early adolescent bipolar disorder phenotype. Am J Psychiatry, 159(6), 927-933.

Geller, B., Tillman, R., Bolhofner, K., \& Zimerman, B. (2008). Child bipolar I disorder: prospective continuity with adult bipolar I disorder; characteristics of second and third episodes; predictors of 8-year outcome. Arch Gen Psychiatry, 65(10), 1125-1133. doi: 10.1001/archpsyc.65.10.1125

Geller, B., Tillman, R., Craney, J. L., \& Bolhofner, K. (2004). Four-year prospective outcome and natural history of mania in children with a prepubertal and early adolescent bipolar disorder phenotype. Arch Gen Psychiatry, 61(5), 459-467. doi: 10.1001/archpsyc.61.5.459

Geller, B., Zimerman, B., Williams, M., Bolhofner, K., Craney, J. L., DelBello, M. P., \& Soutullo, C. (2001). Reliability of the Washington University in St. Louis Kiddie Schedule for Affective Disorders and Schizophrenia (WASHU-KSADS) mania and rapid cycling sections. J Am Acad Child Adolesc Psychiatry, 40(4), 450-455. doi: 10.1097/00004583-200104000-00014

Goldstein, T. R., Axelson, D. A., Birmaher, B., \& Brent, D. A. (2007). Dialectical behavior therapy for adolescents with bipolar disorder: a 1-year open trial. J Am Acad Child Adolesc Psychiatry, 46(7), 820-830. doi:

10.1097/chi.0b013e31805c1613 
Goldstein, T. R., Birmaher, B., Axelson, D., Goldstein, B. I., Gill, M. K., EspositoSmythers, C., ... Keller, M. (2009). Psychosocial functioning among bipolar youth. J Affect Disord, 114(1-3), 174-183. doi: 10.1016/j.jad.2008.07.001

Hamilton, M. (1967). Development of a rating scale for primary depressive illness. Br J Soc Clin Psychol, 6(4), 278-296.

Kaufman, A.S., Kaufman, N.L. . (2004). Kaufman Brief Intelligence Test-Second Edition (KBIT-2). Circle Pines. MN: American Guidance Service.

McCubbin, H.I., \& Thompson, A.I. (1991). Family Assessment Inventories for Research and Practice. . University of Wisconsin-Madison, Center for Excellence in Family Studies: Madison, WI.

Miklowitz, D. J. (2004). The role of family systems in severe and recurrent psychiatric disorders: a developmental psychopathology view. Dev Psychopathol, 16(3), 667-688.

Miklowitz, D. J. (2007). The Role of the Family in the Course and Treatment of Bipolar Disorder. Curr Dir Psychol Sci, 16(4), 192-196. doi: $10.1111 / \mathrm{j} .1467-8721.2007 .00502 . x$

Miklowitz, D. J., Axelson, D. A., Birmaher, B., George, E. L., Taylor, D. O., Schneck, C. D., . . Brent, D. A. (2008). Family-focused treatment for adolescents with bipolar disorder: results of a 2-year randomized trial. Arch Gen Psychiatry, 65(9), 1053-1061. doi: 10.1001/archpsyc.65.9.1053 Miklowitz, D. J., Axelson, D. A., George, E. L., Taylor, D. O., Schneck, C. D., Sullivan, A. E., . . Birmaher, B. (2009). Expressed emotion moderates the 
effects of family-focused treatment for bipolar adolescents. J Am Acad Child Adolesc Psychiatry, 48(6), 643-651. doi:

10.1097/CHI.0b013e3181a0ab9d

Miklowitz, D. J., Biuckians, A., \& Richards, J. A. (2006). Early-onset bipolar disorder: a family treatment perspective. Dev Psychopathol, 18(4), 12471265. doi: $10.1017 / S 0954579406060603$

Neiderhiser, J. M., Reiss, D., Hetherington, E. M., \& Plomin, R. (1999). Relationships between parenting and adolescent adjustment over time: genetic and environmental contributions. Dev Psychol, 35(3), 680-692.

Pavuluri, M. (Unpublished). Childrens Bipolar Depression Rating Scale: A Parent Report Measure.

Pavuluri, M. N., Henry, D. B., Devineni, B., Carbray, J. A., \& Birmaher, B. (2006). Child mania rating scale: development, reliability, and validity. J Am Acad Child Adolesc Psychiatry, 45(5), 550-560. doi:

10.1097/01.chi.0000205700.40700.50

Post, R.M. . (1999). Early recognition and treatment of schizophrenia and bipolar disorder in children and adolescents. . Bipolar Network News, 5, 3-11.

Poznanski, E. . (1984). The Children's Depression Rating Scale - Revised.

Chicago, IL: Rush-Presbyterian Hospital, Department of Psychiatry.

Poznanski, E. O., Cook, S. C., \& Carroll, B. J. (1979). A depression rating scale for children. Pediatrics, 64(4), 442-450.

Robertson, H. A., Kutcher, S. P., Bird, D., \& Grasswick, L. (2001). Impact of early onset bipolar disorder on family functioning: adolescents' perceptions of 
family dynamics, communication, and problems. J Affect Disord, 66(1), 2537.

Schenkel, L. S., West, A. E., Harral, E. M., Patel, N. B., \& Pavuluri, M. N. (2008). Parent-child interactions in pediatric bipolar disorder. J Clin Psychol, 64(4), 422-437. doi: 10.1002/jclp.20470

Sullivan, A. E., Judd, C. M., Axelson, D. A., \& Miklowitz, D. J. (2012). Family functioning and the course of adolescent bipolar disorder. Behav Ther, 43(4), 837-847. doi: 10.1016/j.beth.2012.04.005

Sullivan, A. E., \& Miklowitz, D. J. (2010). Family functioning among adolescents with bipolar disorder. J Fam Psychol, 24(1), 60-67. doi: 10.1037/a0018183

Townsend, L. D., Demeter, C. A., Youngstrom, E., Drotar, D., \& Findling, R. L. (2007). Family conflict moderates response to pharmacological intervention in pediatric bipolar disorder. J Child Adolesc Psychopharmacol, 17(6), 843-852. doi: 10.1089/cap.2007.0046

Washburn, J. J., West, A. E., \& Heil, J. A. (2011). Treatment of Pediatric Bipolar Disorder: A Review. Minerva Psichiatr, 52(1), 21-35.

West, A. E., Jacobs, R. H., Westerholm, R., Lee, A., Carbray, J., Heidenreich, J., \& Pavuluri, M. N. (2009). Child and family-focused cognitive-behavioral therapy for pediatric bipolar disorder: pilot study of group treatment format. J Can Acad Child Adolesc Psychiatry, 18(3), 239-246.

West, A. E., \& Pavuluri, M. N. (2009). Psychosocial treatments for childhood and adolescent bipolar disorder. Child Adolesc Psychiatr Clin N Am, 18(2), 471-482, x-xi. doi: 10.1016/j.chc.2008.11.009 
West, A. E., \& Weinstein, S. M. (2012). A family-based psychosocial treatment model. The Israel journal of psychiatry and related sciences, 49(2), 86-93.

Young, R. C., Biggs, J. T., Ziegler, V. E., \& Meyer, D. A. . (1978). A rating scale for mania: reliability, validity and sensitivity. Br J Psychiatry, 133, 429-435.

Youngstrom, E. A., Danielson, C. K., Findling, R. L., Gracious, B. L., \& Calabrese, J. R. (2002). Factor structure of the Young Mania Rating Scale for use with youths ages 5 to 17 years. J Clin Child Adolesc Psychol, 31(4), 567-572. doi: 10.1207/S15374424JCCP3104_15

Youngstrom, E. A., Findling, R. L., \& Calabrese, J. R. (2004). Effects of adolescent manic symptoms on agreement between youth, parent, and teacher ratings of behavior problems. J Affect Disord, 82 Suppl 1, S5-S16. doi: 10.1016/j.jad.2004.05.016

Youngstrom, E. A., Youngstrom, J. K., Freeman, A. J., De Los Reyes, A., Feeny, N. C., \& Findling, R. L. (2011). Informants are not all equal: predictors and correlates of clinician judgments about caregiver and youth credibility. $J$ Child Adolesc Psychopharmacol, 21(5), 407-415. doi:

10.1089/cap.2011.0032 


\section{VITA}

NAME: $\quad$ Amy T. Peters

EDUCATION: $\quad$ B.A., Psychology, Boston College, Chestnut Hill, MA, 2010

M.A., Psychology, University of Illinois at Chicago, Chicago, IL, 2013

TEACHING: $\quad$ Teaching Assistant, Department of Psychology, University of Illinois at Chicago, Chicago, IL, 2013

Teaching Assistant, School of Education, Boston College, Chestnut Hill, MA, 2008

HONORS: $\quad 2013$ UIC Graduate Student Council Travel Award (\$275) 2013 UIC Psychology Department Travel Award (\$300) 2013 UIC College of Liberal Arts and Sciences Travel Award $(\$ 500)$

2013 UIC Graduate Student College Travel Award (\$375)

2009 Psychology Departmental Grant $(\$ 3,000)$ to fund Thesis

2008 - 2010 Departmental Honors in Psychology (Boston College)

2006 - 2010 University Honors (Boston College)

PROFESSIONAL MEMBERSHIP:

Society for Clinical Child and Adolescent Psychology Association for Behavioral and Cognitive Therapies

PUBLICATIONS: $\quad$ Deckersbach, T., Peters, A.T., Sylvia, L.G., Urdahl, A., Vieira da Silva, P., Otto, M.W., Frank, E., Miklowitz, D., Berk, M., Kinrys, G., and Nierenberg, A.A.: Do co-morbid anxiety disorders moderate the effects of psychotherapy for bipolar disorder? Results from STEP-BD in American Journal of Psychiatry. In press

Sachs, G.S., Peters, A.T. Sylvia, L.G., and Grunze, H.: Polypharmacy and bipolar disorder: What's personality got to do with it? in International Journal of Neuropsychoparmacology. In press.

Sylvia, L.G., Peters, A.T., Deckersbach, T. and Nierenberg, A.A.: Nutrient-based therapies for bipolar disorder. A systematic review in Psychotherapy \& Psychosomatics. 122: 10-9, 2013. 
McMurrich, S., Sylvia, L.G., Dupuy, J.M., Peckham, A.D., Peters, A.T, and Perlis, R.H.: Course, outcomes, and psychosocial interventions for first-episode mania in Bipolar Disorders. 14: 797-808, 2012.

Peters, A.T. and Nierenberg, A.A.: Stepping back to step forward: What lessons do we take from STEP-BD? in Journal of Clinical Psychiatry. 72: 1-3, 2011.

Peters, A.T., Nierenberg, A.A., and Deckersbach, T.: A 32year-old male with mania, leg pain, migraines in Psychiatric Annals. 41: 363-6, 2011.

West, A.E. and Peters, A.T. Bipolar disorders. In Alfano, C., \& Beidel, D. (Eds.) Comprehensive evidence-based interventions for school-aged children and adolescents. Hoboken, NJ: John Wiley \& Sons, Inc. In press. 